\title{
La transformación de la solidaridad familiar desde los servicios sociales básicos en España
}

\section{Sergio Sánchez Castiñeira}

Universidad de Barcelona. Facultad de Educación. Unidad de Formación e Investigación

en Trabajo Social

ssanchezc@ub.edu

https://orcid.org/0000-0001-7568-2462

Recepción: 26-10-2019

Aceptación: 27-02-2020

Publicación: 20-11-2020

\section{Resumen}

Este artículo analiza cómo la práctica cotidiana de los servicios sociales básicos reconstituye las formas de solidaridad familiar dentro de un contexto más amplio de individualización de las relaciones familiares y de transformación socioeconómica. Se trata de un estudio de caso basado en 17 entrevistas y 8 grupos focales que se han realizado a las trabajadoras y educadoras sociales que implementan la asistencia social pública en Tarragona. La extensión y la intensidad de la crisis económica iniciada en 2008 ha evidenciado que una intervención institucional que delega la protección económica en la familia extensa puede resultar ineficaz y saturar las redes familiares. De esta forma, el familismo económico, que todavía es dominante en los servicios sociales, convive con otras prácticas emergentes de solidaridad familiar. En efecto, las profesionales tratan a menudo de potenciar que la familia o la comunidad ayuden a los hogares empobrecidos a través de apoyos afectivos y, en ocasiones, de intercambio de favores (familismo relacional). Asimismo, desde servicios sociales se puede tratar de intervenir contra las situaciones en las que la autonomía y el bienestar de las usuarias están en riesgo debido a que dependen económicamente de los familiares (contrafamilismo). Este artículo concluye que los servicios sociales básicos deben configurarse, por una parte, como capital institucional de los hogares empobrecidos para facilitarles el acceso a relaciones igualitarias y de calidad con su entorno familiar y comunitario. Por otra parte, se justifica que este tipo de intervención debe complementarse con el establecimiento de prestaciones económicas garantizadas que cubran las necesidades materiales básicas.

Palabras clave: asistencia social pública; trabajo social; educación social; estado de bienestar en España; sociología de la familia; solidaridad familiar; familismo; capital social 
Abstract. The transformation of family solidarity through basic social services in Spain

This article analyzes how the daily practice in social services frames new forms of family solidarity, taking into account a broader context of individualization of family relations and of socio-economic transformation. The case study is based on 17 interviews and 8 focus groups with social workers who deliver public social assistance in the city of Tarragona. Institutional action in Spain assumes that the extended family should be responsible for supporting low-income households. Nevertheless, the extension and intensity of the recent economic crisis has brought to light that these social policies can be ineffective and also undermine family networks. Although economic familism is still dominant in social services, it coexists with other emerging practices. Social workers may try to help clients to connect with the extended family to benefit from affective support and sometimes the exchange of favors (relational familism). Furthermore, social services might act against situations in which the autonomy and well-being of clients is at risk because they depend economically on family members (contrafamilism). This article concludes that social services at the local level could be partly framed as a type of institutional capital for low-income households to establish fair and quality relations with their family and communitarian environment. Moreover, this type of public action should be accompanied by a guaranteed minimum income.

Keywords: public social assistance; social services; social workers; welfare state in Spain; family; family solidarity; familism
Sumario
1. Introducción
5. Resultados
2. Marco teórico
6. Discusión de resultados
3. Los servicios sociales básicos en la
7. Conclusiones
ciudad de Tarragona
Referencias bibliográficas
4. Método

\section{Introducción}

La pasada crisis económica y fiscal ha exacerbado los procesos de empobrecimiento de las familias con niños en España. Entre los años 2008 y 2018, la tasa de riesgo de pobreza o de exclusión en España ha pasado del 23,8 \% al 26,1 \%, mientras que la tasa de privación material severa se ha incrementado del 3,6 \% al 5,4 \% (Llano, 2019). En este contexto, los niveles autonómico y estatal no han ofrecido respuestas decisivas y han delegado buena parte de la atención al nivel local (González y Pino, 2017). Los servicios sociales de primaria o básicos son los dispositivos de la Administración local que tienen la función de atender las necesidades socioeconómicas y relaciones de la población. Sin embargo, esta asistencia social pública no dispone de recursos suficientes para garantizar las necesidades socioeconómicas más básicas (Aguilar et al., 2012). Las prestaciones públicas son escasas y fragmentadas, y deben integrarse con otros recursos provenientes de las transferencias intrafamiliares (Arriba y Moreno, 2005). En última 
instancia, las intervenciones institucionales contra la pobreza toman un papel residual porque la familia extensa se considera la principal fuente de protección social en los sistemas de bienestar del sur de Europa (Paugam, 2005).

En efecto, las redes familiares han amortiguado algunos de los efectos de la crisis económica (Martínez, 2014). Sin embargo, la reciente extensión y la intensificación de las necesidades podrían debilitar la solidaridad familiar en España (Marí-Klose, 2016). Asimismo, los procesos globales de mercantilización e individualización están reconfigurando los mecanismos de reciprocidad de las familias en las últimas décadas (Déchaux, 2007; Meil, 2011). Este contexto acentúa la ineficacia de la asistencia social pública, que debe orientar a los hogares empobrecidos hacia los recursos de la familia extensa o de la comunidad para poder atender las situaciones de mayor riesgo de exclusión social (Sánchez, 2019a). En realidad, este tipo de política social podría resultar «reconstitutiva» de las desigualdades sociales (Adelantado y Noguera, 1999), porque exprime los recursos de unos sistemas familiares que se encuentran flanqueados de dificultades económicas. No obstante, la pobreza es un fenómeno económico, relacional y simbólico (Lister, 2004) y, por tanto, los servicios sociales intervienen de forma personalizada sobre diferentes aspectos de la vida de las personas. Además, el producto final de las políticas sociales es, en parte, indeterminando, pues depende de cómo las profesionales aplican los principios y las normativas a unas circunstancias locales y organizativas específicas (van Berkel y van der Aa,; 2012).

El presente artículo muestra las nuevas estrategias familistas que se despliegan desde primera línea de los servicios sociales básicos de Tarragona para reconstituir las relaciones familiares en un recurso de apoyo efectivo, de acuerdo con un contexto más amplio de transformación de la institución familiar. De esta forma, se identifican algunas de las nuevas funciones y responsabilidades que la familia podría adquirir en el sistema de bienestar español. Este artículo presenta un marco teórico sobre el papel de las relaciones familiares y comunitarias en los estados de bienestar del sur de Europa. Más concretamente, se fundamenta el papel activo de los servicios sociales básicos en la configuración de las nuevas formas de solidaridad en estos contextos de transformaciones socioeconómicas y culturales. Seguidamente, se describen los servicios sociales en Tarragona y se presentan las estrategias y técnicas de investigación. Los resultados analizan las condiciones que favorecen el predominio del familismo en los servicios sociales básicos. Asimismo, se presentan dos tipos de prácticas institucionales emergentes: las que directamente desafían el familismo económico y las que se centran en la vertiente relacional de la solidaridad familiar. Finalmente, se discuten los resultados y se presentan las conclusiones.

\section{Marco teórico}

\subsection{El estado de bienestar en el sur de Europa}

Durante la Revolución Industrial, las funciones de producción se externalizaron de la familia, al mismo tiempo que la acción estatal reconfiguró los vínculos familiares en términos de solidaridad y de protección social (Lenoir, 
2007). Este tipo de apoyo mutuo entre parientes mantiene un mayor apoyo institucional en los países del sur de Europa (Flaquer, 1998). Estos países tienen un gasto social relativamente reducido, al mismo tiempo que muestran una mayor proporción de hogares donde conviven diferentes generaciones y de trasferencias dentro la familia extensa (Comisión Europea, 2008). La familia adquiere prioridad en la escala de valores de la población debido a este contexto socioeconómico e institucional y, al mismo tiempo, esta orientación normativa favorece el mantenimiento del modelo de bienestar familista (Moreno, 2001).

El familismo está fundamentado en un sistema de distribución de recursos y de satisfacción de las necesidades que apela a sentimientos de unidad y de solidaridad basados en el parentesco, el matrimonio o la amistad (Tönnies, 1984). Las diversas intervenciones públicas parten del supuesto de que la familia es la responsable de la protección de sus miembros y de que las políticas deben fomentar la solidaridad entre los parientes (Flaquer, 2004). Este carácter subsidiario de las políticas sociales implica que los derechos sociales no están suficientemente reconocidos (Saraceno, 1995). Asimismo, algunos derechos explicitados pueden diluirse en el momento que deben concretarse. Por ejemplo, la renta mínima de inserción, que la ley había establecido como una prestación garantista, ha pasado a ser una ayuda selectiva y discrecional, debido a que la implementación de esta medida se ha delegado en unas agencias locales de servicios sociales que están infradotadas de recursos humanos y económicos (Aguilar, 2013).

Ciertamente, los vínculos familiares y sociales aportan diversos tipos de beneficios en el aspecto material y en el emocional, al mismo tiempo que reducen los riesgos de desintegración social que pueden producir unas políticas que no tengan en cuenta las relaciones de reciprocidad constituidas (Castel, 1995). El apoyo entre familiares conecta con una experiencia de normalidad que protege la identidad social de las personas empobrecidas. Por el contrario, aquella población que solo dispone de apoyos más formalizados e impersonales padece una situación objetiva de privación mayor, y transmite una sensación más desgarradora de derrota y de exclusión (Sánchez, 2019b).

Sin embargo, las redes familiares y sociales presentan límites importantes como mecanismo principal de protección ante los actuales riesgos de pobreza y de exclusión social. Por una parte, estos sistemas de reciprocidad tienden a excluir la participación de los hogares con menos ingresos económicos de las actividades de ayuda mutua (Williams y Windebank, 2000). Por otra parte, relaciones familiares y comunitarias también pueden impactar de forma desfavorable en las personas empobrecidas ("capital social negativo») (Portes, 1998. Los vínculos familiares y comunitarios pueden representar fuertes presiones hacia la conformidad con las normas y las expectativas del grupo, que no siempre están orientadas al progreso personal o familiar, o a la inclusión social. Asimismo, el hecho de traspasar a las familias la responsabilidad de protección económica acentúa las desigualdades de género, puesto que las mujeres tienden a someterse a mayores restricciones para amortiguar los efectos de la privación material (Ridge, 2010) Además, las mujeres suelen asumir el trabajo 
de canalizar los recursos dentro de la red familiar, así como entre la familia y los diversos servicios del estado de bienestar (Power et al., 2011).

En definitiva, un estado de bienestar que delega la protección social de los individuos a la familia y la comunidad en detrimento del Estado tiene efectos negativos para el bienestar económico general y la igualdad de oportunidades (Esping-Andersen, 1999). De esta manera, países de orientación familista como España, Portugal o Italia han presentado tradicionalmente unos porcentajes altos de hogares con niños en situación de pobreza (OECD, 2011). Además, se produce una mayor transmisión intergeneracional de la pobreza que en los países nórdicos y continentales (Raitano, 2015).

\subsection{Las transformaciones socioeconómicas e ideológicas de la solidaridad familiar}

El familismo en los países del sur de Europa ha demostrado su vigencia ya que constituye un sistema de adaptación social específico a las necesidades del capitalismo avanzado (Flaquer, 2004). No obstante, las profundas trasformaciones de la institución familiar en las últimas décadas han reconfigurado la forma y el contenido de este modelo de bienestar. Las familias son cada vez más extensas desde un punto de vista intergeneracional, pero cuentan con un menor número de miembros dentro de cada generación. Esto favorece los apoyos entre progenitores e hijos adultos, pero también satura la red familiar al disponer de menos parientes (Andreotti et al., 2001). Asimismo, se debilita el carácter estatuario de la solidaridad familiar, que cada vez más requiere que los parientes inviertan los recursos y dispongan de las habilidades sociales para mantener las relaciones familiares (Meil, 2011). Finalmente, la división sexual del trabajo, que había sido fundamental para mantener la solidaridad familiar en el sur de Europa, se ha atenuado debido al mayor nivel educativo de las nuevas generaciones de mujeres y de su acceso al mercado laboral (Flaquer, 2010).

Cambios en las actitudes reflejan las mayores dificultades estructurales de la familia extensa para mantener la solidaridad familiar. Las generaciones más jóvenes tienden a responsabilizar más al Estado en la atención de la infancia $\mathrm{y}$ de adultos dependientes (Bazo, 2012). Asimismo, se produce una mayor «conyugación» de las relaciones familiares, puesto que se incrementa la inclinación a pedir apoyo material y favores a la pareja en detrimento de recurrir a otros familiares (Flaquer, 2010). Por otro lado, las relaciones recíprocas que el núcleo familiar establece con los parientes se fundamentan cada vez más en aspectos electivos (ligados a la mayor individualización y reflexividad) que estatutarios (deberes y derechos recíprocos como tío, prima, madre, etcétera) (Beck et al., 1994). De esta forma, la solidaridad familiar tiende a basarse en la "familia negociada», que reclama un mayor esfuerzo en el mantenimiento de las relaciones (Meil, 2011).

Por último, las nuevas formas de familias se han extendido, lo que ofrece oportunidades de desarrollo personal sobre todo a mujeres y a otros colectivos minorizados. Sin lugar a duda, este cambio representa un avance neto en la 
superación de las desigualdades de género. Sin embargo, la falta de políticas familiares en España incrementa la vulnerabilidad y la inestabilidad de las formaciones familiares que no siguen el modelo de los hogares con dos adultos trabajadores (Esping-Andersen, 2002). Además, a pesar de un aumento de la proporción de mujeres trabajadoras, las mujeres con niveles bajos de estudio o inmigrantes pueden sufrir mayores riesgos de pobreza en el contexto de un mercado laboral segmentado (Parella, 2003).

\subsection{La construcción institucional del familismo desde los servicios sociales}

En las últimas décadas, se han producido cambios en la estructura y las orientaciones normativas de la institución familiar (Flaquer, 2010; Meil, 2011). En España, estas trasformaciones de largo recorrido se interrelacionan con un incremento y una intensificación de las necesidades de la población y con los recortes en las prestaciones del estado de bienestar que se han producido durante la crisis económica. Las políticas contra la pobreza y, más concretamente, los servicios sociales básicos ocupan un lugar central en en la transformación de la solidaridad familiar a través de a unas intervenciones que impactan sobre las diferentes funciones y relaciones de las familias (Saraceno, 2002).

Los servicios sociales básicos están gestionados por los entes locales, pero se configuran en un contexto multinivel, que incluye aspectos legislativos, reglamentarios y de financiación de las administraciones central, autonómica y local. Asimismo, se les atribuyen diferentes funciones, como cubrir las carencias materiales de la población, atender a las personas víctimas de violencia o proteger a la infancia en riesgo de negligencia, maltrato o abuso. ${ }^{1}$ En este sentido, los servicios sociales básicos se constituyen en parte a partir de un conjunto no necesariamente coherente de influencias que provienen del contexto local y supralocal, de las normativas y regulaciones, así como de los diversos aspectos de las organizaciones (que incluyen los recursos disponibles) (Vinzant y Crothers, 1996). No obstante, estas políticas sociales se concretan en última instancia a través de las prácticas de unos actores en primera línea que deben gestionar objetivos a veces contradictorios y la escasez relativa de recursos (Lipsky, 1980).

Por otra parte, los servicios sociales básicos se insertan en un «sistema local de bienestar» que está formado por diversas combinaciones de actores formales e informales, públicos y privados, que están implicados en la provisión de recursos de bienestar (Andreotti et al., 2012). Dentro de este sistema, los servicios sociales básicos toman un rol central por dos motivos. Primero, representan la institución responsable de atender las necesidades socioeconómicas

1. Esta investigación no analiza las intervenciones sociales relativas a la Ley 39/2006 de Promoción de la Autonomía Personal y Atención a las Personas en Situación de Dependencia que los servicios sociales básicos tienen encomendadas. La atención a la dependencia física constituye un universo de intervención relativamente independiente de la atención al resto de necesidades sociales, puesto que estas últimas tienden a compartir una situación de autonomía funcional y de empobrecimiento. 
básicas de la población (Fantova, 2008). En segundo lugar, cuentan con una infraestructura de recursos (prestaciones, servicios, profesionales, oficinas en todos los territorios), con un relativo poder económico (contratan y subvencionan prestaciones y servicios externos) y con unas atribuciones legales (por ejemplo, la definición administrativa de aquellos niños en situación «de riesgo»), que condicionan el funcionamiento de los demás agentes de bienestar del territorio.

\section{Los servicios sociales básicos en la ciudad de Tarragona}

Tarragona es una ciudad de 131.507 habitantes (2017), capital de una de las cuatro provincias de la Comunidad Autónoma de Cataluña (España). La ciudad presenta una media de renta familiar disponible superior a la media de los municipios en Cataluña y en España, pero también sufre unas mayores desigualdades socioeconómicas y menos inversión en políticas sociales. En 2013, la tasa de paro registrado ascendía al $19 \%$ y la tasa de cobertura de prestaciones y subsidios de desempleo era del $50 \%$ (Sánchez, 2019a). ${ }^{2}$ Un contexto local de bienestar especialmente restrictivo como es Tarragona puede producir unas políticas más marcadamente subsidiarias de la familia. De esta forma, el análisis del caso de Tarragona puede facilitar la identificación de las estrategias económicas familistas que los servicios sociales en España deben generar para dar respuesta a las necesidades de la población (Yin, 2014).

\subsection{Servicios sociales básicos}

La Ley 12/2007 de Servicios Sociales del Parlamento catalán encarga a las administraciones locales la organización y la gestión de los servicios sociales básicos, que tienen la responsabilidad de atender las necesidades socioeconómicas y de integración social de la población. Estos servicios están gestionados por trabajadoras y educadoras sociales y se distribuyen por los barrios a través de los «centros de servicios sociales». En la ciudad de Tarragona, los servicios sociales básicos se organizan a partir de cinco centros distribuidos por el territorio, que, en su conjunto, cuentan con 33 trabajadoras sociales y educadoras sociales. ${ }^{3,4}$ Durante los momentos más duros de la crisis económica (año 2013), este personal realizó al menos una intervención con 5.523 personas (el $4,2 \%$ de la población de la ciudad), y tuvieron incidencia en 3.375 hogares (el 6,2\% de los hogares de la ciudad) (Sánchez, en prensa). Con relación a la

2. La tasa de desempleo total (registrado y no registrado) que recogía la EPA para toda la provincia ascendía al $26 \%$ (no se dispone de este dato para la ciudad de Tarragona).

3. Este artículo emplea el femenino como genérico ya que la inmensa mayoría de profesionales y personas usuarias de los servicios sociales se definen como mujeres.

4. Estas 33 profesionales pueden trabajar a tiempo parcial o a tiempo completo, y equivalen a 26 profesionales que trabajasen a tiempo completo. Estas cifras no incluyen al personal de servicios sociales básicos destinado a atender las situaciones de dependencia física severa (ver nota 1 ). 
media de las otras ciudades de Cataluña de tamaño similar, lo servicios sociales básicos de Tarragona disponían de menos recursos y debían hacer frente a un contexto con mayores desigualdades y más infradotado de prestaciones de las administraciones central y autonómica (Sánchez, en prensa).

La principal medida contra la pobreza que los servicios sociales gestionaban era la prestación económica de urgencia social («ayuda de urgencia»), destinada a sufragar un gasto básico puntual de los hogares. Durante la crisis económica, estas prestaciones se habían convertido en la principal medida en el ámbito local para paliar las situaciones de desamparo económico causadas por los recortes en las rentas mínimas por parte de la Administración autonómica (ECAS, 2012). La Ley 12/2007 de Servicios Sociales del Parlamento catalán establecía que las prestaciones (económicas, de servicios y tecnológicas) eran un «derecho subjetivo», pero que su tramitación debía acreditar que el hogar tenía una «necesidad». En tanto que no se llegó a formalizar una definición operativa de "necesidad», quedaba a discreción del personal (y a la capacidad del servicio) la decisión sobre qué se consideraba una necesidad, y si esta necesidad debía ser cubierta desde la esfera familiar o comunitaria. Igualmente, la capacidad de distribuir estas prestaciones en función de las necesidades reales de la población se encontraba limitada porque la regulación autonómica las supeditaba a la disponibilidad presupuestaria. ${ }^{5}$

Por otra parte, los servicios sociales de Tarragona disponían de un «reglamento interno» de las prestaciones de urgencia que había sido elaborado por los cuadros intermedios de la organización (anteriores trabajadoras o educadoras sociales en primera línea de intervención). Este documento establecía que el objetivo de estas prestaciones era "procurar cubrir las necesidades más básicas de las personas y/o familias, sobre todo de alimentación y de suministros» (IMSST, 2014). ${ }^{6}$ Establecía un límite de ingresos para el acceso que era más alto que el de otras prestaciones como la renta mínima de inserción: 664 mensuales para 2 personas (correspondiente al indicador de renta de suficiencia de Cataluña en 2014). Este documento establecía como criterio de selección de beneficiarios más específico que el hogar "disponga de una red familiar de apoyo que pueda garantizar el gasto en aquella necesidad». Por último, este documento no se hacía público ni se mostraba a las usuarias demandantes.

Finalmente, los servicios sociales básicos se organizaban en equipos funcionales según los siguientes ámbitos de intervención: situaciones puntuales o moderadas de privación o crisis familiar, trayectorias acentuadas de exclusión social, violencia machista y protección a la infancia. A pesar de esta diferenciación de las usuarias, la mayoría de las usuarias de los servicios sociales básicos estaban empobrecidas y, por tanto, la acción de los servicios sociales básicos comportaba siempre la gestión de una situación condicionada y deformada por las desigualdades económicas. Esto implicaba que las intervenciones con

5. Ley 13/2006 de prestaciones sociales de carácter económico del Parlamento catalán.

6. Traducción propia del catalán. 
las usuarias entremezclasen las medidas socioeconómicas con otras orientadas a capacitar a las usuarias en sus relaciones familiares.

En definitiva, los servicios sociales básicos en Tarragona se encontraban durante la crisis en un contexto socioeconómico e institucional con escasos recursos para atender las necesidades económicas de las familias. Asimismo, el marco legislativo y organizativo proporcionaba a las profesionales un cierto margen de decisión al mismo tiempo que orientaba su práctica para que la (inevitable) selección de las familias beneficiarias de las prestaciones se realizase sobre principios familistas.

\section{Método}

El estudio se basa sobre todo en 17 entrevistas individuales y en 8 grupos focales con personal de los servicios sociales básicos que fueron realizadas, grabadas, transcritas y analizadas por el investigador. Estas técnicas de investigación resultan pertinentes en este estudio porque permiten obtener una descripción e interpretación de las prácticas cotidianas del personal de los servicios sociales básicos y, de esta forma, comprender el funcionamiento de esta institución (Bertaux, 2010). Los dilemas éticos y las tensiones emocionales que conlleva la atención cotidiana de situaciones de miseria y desesperación conllevan que el personal en primera línea de intervención desarrolle un alto grado de reflexividad sobre su quehacer cotidiano. Además, las formas preestablecidas de intervención en servicios sociales requieren que este personal desarrolle una enorme habilidad en el manejo de la palabra y en la dinámica de la entrevista. Esto hace de la entrevista semiestructurada la técnica más adecuada para identificar las diferentes circunstancias que se entretejen en esta intervención institucional (Sánchez, en prensa).

El personal de servicios sociales finalmente entrevistado se ha seleccionado de forma intencional y no estadística. Este tipo de muestra pretende identificar la máxima variabilidad de circunstancias y experiencias en un ámbito institucional específico (purposive sampling) (Silverman, 2010). De esta manera, se ha pretendido entrevistar a un número significativo de personal tanto de zonas del centro de la ciudad (Part Alta y Part Baixa) como de la periferia (Ponent, Sant Salvador y Sant Pere i Sant Pau). Esta fragmentación territorial forma parte del imaginario de la población, y al mismo tiempo también refleja una desigualdad socioeconómica en favor del centro, así como un funcionamiento más masificado en los servicios sociales básicos de la periferia (Sánchez, en prensa). Igualmente, se ha pretendido obtener una muestra diversa en función de las diferentes especializaciones dentro de los servicios sociales (equipos de Primera Acogida, de Inclusión de Familias, de Violencia de Género y Socioeducativo de Infancia), además de incluir a las coordinadoras y directora técnica (tabla 1).

El análisis de los relatos de las profesionales se realizó a través del programa Atlas.Ti7, a partir de un enfoque inductivo que generaba nuevos conceptos en interacción con listas temáticas que se habían elaborado previamente. Las entrevistas individuales tuvieron lugar en el verano de 2015 y duraron una 
Tabla 1. Personal colaborador directo de la investigación.

\begin{tabular}{|c|c|c|c|c|c|}
\hline Género y edad & Zona & Equipo & $\begin{array}{l}\text { Entrevista } \\
\text { individual }\end{array}$ & $\begin{array}{l}\text { Grupo } \\
\text { focal } 1\end{array}$ & $\begin{array}{r}\text { Grupo } \\
\text { focal } 2\end{array}$ \\
\hline Mujer, 43 & Ponent & Inclusión & $x$ & $x$ & $x$ \\
\hline Mujer, 31 & Part Baixa & Inclusión & $x$ & & \\
\hline Mujer, 44 & Part Baixa & Socioeducativo & $x$ & & \\
\hline Hombre, 56 & Ponent & Socioeducativo & $x$ & $x$ & $x$ \\
\hline Mujer, 35 & Ponent & Acogida & $x$ & $x$ & $x$ \\
\hline Mujer, 47 & Part Baixa & Acogida & $x$ & $x$ & $x$ \\
\hline Mujer, 34 & Ponent & Acogida & $x$ & $x$ & $x$ \\
\hline Mujer, 40 & Ponent & Acogida & $x$ & $x$ & $x$ \\
\hline Hombre, 38 & Ponent & Socioeducativo & $x$ & $x$ & $x$ \\
\hline Mujer, 37 & Part Alta & Socioeducativo & $x$ & & \\
\hline Hombre, 41 & Ponent & Inclusión & $x$ & $x$ & $x$ \\
\hline Mujer, 28 & Ponent & Violencia de Género & $x$ & & \\
\hline Mujer, 34 & Part Alta & Socioeducativo & $x$ & $x$ & $x$ \\
\hline Mujer, 36 & Ponent & Violencia de Género & $x$ & $x$ & $x$ \\
\hline Hombre, 44 & Part Alta & Socioeducativo & $x$ & $x$ & $x$ \\
\hline Mujer, 41 & Part Baixa & Socioeducativo & $x$ & & \\
\hline Hombre, 48 & Ponent & Socioeducativo & & $x$ & $x$ \\
\hline Mujer, 56 & Sant Pere & Acogida & & $x$ & $x$ \\
\hline Mujer, 53 & Sant Salvador & Inclusión / Acogida & & $x$ & $x$ \\
\hline Hombre, 42 & Ponent & Socioeducativo & & $x$ & $x$ \\
\hline Mujer, 47 & Part Alta & Socioeducativo & & $x$ & $x$ \\
\hline Mujer, 29 & Ponent & Socioeducativo & & $\mathrm{x}$ & $x$ \\
\hline Mujer, 37 & Sant Pere & Inclusión & & $x$ & $x$ \\
\hline Mujer, 31 & Ponent & Inclusión & & $x$ & $x$ \\
\hline Mujer, 61 & Ciudad & Violencia de Género (C) & & $x$ & $x$ \\
\hline Mujer, 62 & Ciudad & Directora técnica & & $x$ & $x$ \\
\hline Mujer, 46 & Ciudad & Discapacidades (C) & & $x$ & $x$ \\
\hline Mujer, 46 & Ciudad & Inclusión (C) & & $x$ & $x$ \\
\hline Mujer, 42 & Ciudad & Socioeducativo (C) & & $x$ & $x$ \\
\hline Mujer, 60 & Ciudad & Protección a Infancia (C) & & $x$ & $x$ \\
\hline Hombre, 63 & Ciudad & Tercera Edad (C) & & $x$ & $x$ \\
\hline
\end{tabular}

Fuente: elaboración propia.

media de 80 minutos. El análisis se orientó a la identificación y explicación de las diferentes situaciones de dificultades económicas que se presentaban en los servicios sociales, y de las estrategias que las profesionales empleaban para darles respuesta. En abril de 2016, se llevaron a cabo ocho grupos focales, que duraron un promedio de 75 minutos. Cada una de las 28 trabajadoras y educadoras sociales participantes intervino en dos grupos focales durante una misma mañana. El primer grupo de discusión trató sobre los recursos para la intervención social que la propia organización podía proporcionar. El 
segundo grupo exploró el uso que las usuarias hacían (o no) de las fuentes de bienestar informales, como por ejemplo de la familia. Durante el transcurso de los grupos, diversos extractos de las entrevistas individuales fueron leídos para clarificar o desarrollar los resultados que se habían extraído inicialmente de las entrevistas individuales (Morgan, 1996).

\section{Resultados}

\subsection{La orientación familista en la asistencia social pública}

En contraposición con otras formas tradicionales de caridad, la asistencia social pública se define como un derecho que la ciudadanía puede exigir (Fantova, 2008). Sin embargo, la insuficiencia de recursos de los servicios sociales implica que los instrumentos garantistas que se han diseñado inicialmente quedan desfigurados durante el proceso de implementación. La siguiente cita muestra cómo la realidad cotidiana de estos servicios requiere que la adjudicación de prestaciones se fundamente en las apreciaciones del personal, con detrimento de los derechos sustantivos y procedimentales de las usuarias (Lister, 2004):

Yo sé que este reglamento dice que una familia puede disponer de ayudas de urgencias anuales de hasta 800 euros. Esto la gente no lo sabe, sino me parece que tendríamos colas de familias exigiendo los 800 euros. [...] Más que el reglamento, yo utilizo mi criterio. (Joana, E. I.)

El «criterio» de las profesionales en este marco familista a menudo presupone que los hogares empobrecidos tienen a su alcance unos recursos familiares y comunitarios que deben ser movilizados:

Te vienen con la historia «estoy solo». Pero luego vas rascando y siempre hay alguien, siempre hay familia, y si no la familia, hay la comunidad. Les dices: «Debes un mes de alquiler. ¿Qué ha podido pasar? ¿Quién te puede ayudar?». $\mathrm{Y}$ te dicen: «Pues mira, no tengo a nadie». O: «Tengo a mi madre, pero no le puedo pedir porque este mes me ha ayudado mucho». Entonces, quizás le haces la ayuda, pero es importante que ellos tomen conciencia de que la ayuda no es para siempre, de que es importante que ellos rasquen de su propia red. (Alba, E. I.)

La cita anterior muestra que una de las funciones implícitas de los servicios sociales es que las propias usuarias interioricen que las prestaciones de asistencia social son un recurso subsidiario del apoyo familiar. De esta manera, los escasos recursos institucionales tienden a maximizarse porque se destinan a las usuarias más excluidas y desposeídas:

La usuaria me dice: «¿Tengo que volver con mi hermano si también lo está pasando mal?». Y yo les digo: «Pues sí. Es que es un recurso, es que si ellos [el hermano y su núcleo familiar] tienen un techo y te puede dejar una habitación...». Es que jolines, antes de generar y gestionar un recurso... Que igual 
habrá otras personas que sí estarán con mucha más urgencia, porque no habrá familia extensa. (Rosario)

Por otro lado, los servicios sociales se enfrentan a necesidades derivadas de una situación de desempleo o de vulnerabilidad residencial estructural, las cuales perduran en el tiempo. No obstante, la asistencia social pública solamente puede otorgarse de forma puntual. Seguramente, esta característica de las prestaciones es otro factor que condicionan para que los servicios sociales deleguen la responsabilidad de protección social en la solidaridad familiar. Como muestra la siguiente cita, un sistema asistencia social basado en prestaciones puntuales deja desemparadas a aquellas usuarias empobrecidas que carecen de apoyo familiar:

Se establecen límites entre el tiempo de las ayudas, entre una ayuda y otra. Pero claro, es que vemos situaciones muy extremas. Yo, como técnica, igual le tramitaría a esta familia cada mes. Porque cada mes comen, pero, claro, no puedo. (Joana, E. I.)

Los procesos de nuclearización de las familias que se han producido en las últimas décadas (Flaquer, 2010) incrementan el riesgo de privación severa de muchos hogares empobrecidos en el contexto de bienestar español. En este caso, los servicios sociales deben primero promover la solidaridad de los parientes para posteriormente poder delegarles la responsabilidad de protección económica de los hogares precarizados. Este proceso de restablecimiento de los vínculos familiares para obtener recursos materiales requiere un personal que despliegue un complejo «trabajo emocional» (Sieben y Wettergren, 2010). Asimismo, la organización debe facilitar espacios y tiempos específicos para atender a las usuarias de forma individual y confidencial.

No quiero decirles que deben hablar con ellos [los parientes]. [...] Tenía el caso de una familia con un bebé; los iban a desahuciar. Tenían familia en Extremadura y no les querían llamar. Yo, sin forzar, pero sí les decía: «Es normal que la relación se deteriore, pero si ellos saben que lo estáis pasando mal, pues quizás os quieran ayudar». Y, efectivamente, cuando al final se deciden a llamar, los parientes les dicen que podían disponer de la casa de los abuelos en el pueblo, que estaba cerrada. Porque ella decía: «Yo no puedo pedirles nada». Y yo les decía: «No pidas, explica tu situación». Yo les ayudo a buscar la manera de buscar ayuda, si aquel hombre me decía que no se atrevía a decir a su familia que se iba a quedar en la calle, yo le digo: «A lo mejor lo que tienes que hacer, ahora que llega la Navidad, es llamar, felicitar, explicarles que tu situación es apurada». [...] En realidad, el espacio de entrevista [con la usuaria] es mi mejor recurso. (Leticia)

El número de personas jóvenes emancipadas menores de 30 años ha descendido durante la crisis económica en España (OBJOVI, 2013). Los servicios sociales básicos contribuyen a este fenómeno a través de la configuración de hogares multigeneracionales que puedan maximizar la solidaridad familiar. 
La falta de recursos públicos para la emancipación de la juventud (Moreno Mínguez, 2012) y para atender las necesidades de las familias monoparentales (Flaquer et al., 2006) limita las opciones familiares reales de madres jóvenes. En estos casos, se puede considerar que lo más adecuado para prevenir el riesgo de pobreza y de exclusión social es disuadir a la usuaria de su intención de formar un hogar independiente de la familia extensa. El personal puede entonces movilizar una definición infantilizante de la usuaria, posiblemente para que su intervención no entre en conflicto con los objetivos manifiestos de libertad y capacitación que definen las profesiones que implementan los servicios sociales (CGTS, 2012). En la siguiente cita, la profesional describe esta práctica de forma expresionista:

Hicimos una entrevista disuasiva, en el sentido de: «Lárgate de aquí, no hay ayudas para mamás que se han ido de casa de sus padres porque, con 17 años, consideraban que el ambiente era tenso. No, no tenemos esta ayuda. Lo siento». A ver, es que no tenía que haberse ido de casa de sus padres. Con 17 años y embarazada, lo que tenía que hacer es acogerse a la ayuda de los padres de mantenerla a ella y al niño en casa. Y espabilarse ella. Y que estudie, que busque curro. Eso es lo correcto. (Beatriz, E. I.)

Ciertamente, la utilización del humor y de la despersonalización puede ser una estrategia adaptativa en contextos donde el personal debe enfrentarse a dramas humanos sin la capacidad de darles respuesta (Becker et al., 1961).

Ante la escasez de políticas sociales y familiares, esta estrategia familista de los servicios sociales puede ser eficaz para proteger a las familias jóvenes de la privación material extrema, pero a cambio de sacrificar sus proyectos personales y familiares y de incrementar las tensiones familiares (Lasheras et al., 2012) y. Además, la legitimización de este tipo de intervención puede conducir a una crítica severa de la usuaria, y convertir así una intervención social de ayuda en una relación social degradante (Paugam, 2002). Por otra parte, un contexto organizativo donde las profesionalaes disponen de escaso tiempo para establecer relaciones de confianza con las usuarias incrementa la ineficacia de tener que aplicar preferentemente la expectativa familista. La siguiente cita expone de forma preocupante cómo el supuesto familista puede prevalecer incluso en situaciones donde no se ha podido dirimir si los parientes podrán cubrir las necesidades más elementales de una familia monoparental.

(Respecto a una mujer marroquí embarazada) El marido nunca ha vivió aquí, es ella la que ha ido bajando [a Marruecos] de vez en cuando. Y vive aquí con los padres y el hermano. Me dice que el padre no la quiere ayudar y que ella no tiene dinero. Ella dice: «Y tengo dos hijos, ¿̇cómo trabajo?». [...] En este caso, por muy mal que te sepa, no se le ha hecho el pirmi [renta mínima de inserción], solo una ayuda puntual de Cruz Roja. Ella vive en casa de los padres, el marido no está aquí y tampoco sabes si aporta o no aporta, dicen que no trabaja, pero ¿̨tú qué sabes? (Laura, E. I.) 
Por otro lado, la tendencia institucional a disolver las fronteras económicas entre el núcleo familiar y la familia extensa puede intensificarse cuando se identifican algunas usuarias con determinadas identidades culturales («el otro») (Izaola y Zubero, 2015). La siguiente cita muestra que el prejuicio de que los parientes mantengan intensas relaciones de ayuda mutua puede intensificarse sobre determinados colectivos que son especialmente vulnerables:

Había el caso de una familia [gitana] que la nena no iba por las tardes porque la familia no podía pagar la parte [del comedor escolar] que no cubría nuestra beca. Entonces se achuchó a la familia para que entre todos, que son un montón, pusieran lo que faltaba. Claro, si el abuelo, que es el que se encarga [de la nena], no puede asumirlo, pues también hay tíos que llevan los niños al mismo colegio y que se pueden encargar. Él me decía: «iPero es que claro, yo no quiero pedir nada a mis hermanos!». Y yo le respondía: «Pero, a ver, sois familia, ¿no?». Es una cosa que hemos trabajado juntamente con la escuela. (Carla, E. I.)

Los servicios sociales básicos se despliegan sobre una «lógica de proximidad» al territorio (Subirats, 2007), donde las relaciones que las profesionales establecen con otros actores de la zona (usuarias, agencias, etcétera) permiten conocer las relaciones familiares de la usuaria. En un contexto familista, esta faceta del «trabajo en red» puede suponer un riesgo para la capacidad de decisión (y la privacidad) de las usuarias. Por otra parte, los supuestos familistas también se pueden proyectar entre los compatriotas, o entre los miembros de una supuesta comunidad:

Tengo unos [usuarios] chilenos que vinieron solos, sin familias, pero que se han juntado con otros. Entre unos y otros se ayudan. Son su familia en España. Quizás, económicamente, dinero físico, no [se dan], pero sí que se dicen: «Te compro la compra del fin de semana»; "Te doy el plato de comida de tu hija, o «Vamos a comprarnos ropa». Para mí eso ya es una ayuda. La gente tiene red y son gente superviviente, saben cómo buscarse la vida. Lo que pasa que te tienen que vender la moto. Evito hacer siempre ayudas, prefiero enseñar a la gente cómo buscar trabajo o cómo buscarse la vida. (Alba, E. I.)

En este caso, la institución omite que las actitudes y las capacidades de los inmigrantes para formar parte de las redes sociales varían en función de las trayectorias de inmigración y de los grupos socioculturales (McCave et al., 2013). Asimismo, la expectativa institucional de la norma de la reciprocidad entre compatriotas o vecinos conlleva el riesgo de generar relaciones de intercambio más descarnadamente contractuales, puesto que carecen de la protección que tienden a incorporar los vínculos afectivos entre familiares. De esta forma, hay usuarias de servicios sociales de Tarragona que inicialmente debieron recurrir a la ayuda de compatriotas y terminaron sufriendo procesos severos de endeudamiento (Sánchez, 2019b).

El familismo de los servicios sociales también se canaliza a partir de los supuestos de que, en determinadas ocasiones, las prestaciones económicas pue- 
den ser utilizadas de forma errónea o abusiva, y de que existe una población que «depende» de las «ayudas» de los servicios sociales (Ivalua, 2016). En casos extremos, unos servicios sociales de orientación familista pueden permitir que la profesional desvincule las prestaciones económicas de las funciones de la organización:

Considero que no debemos tramitar los «vales de alimentación» [tarjetas tipo visa que las usuarias pueden utilizar en supermercados ordinarios]. Nosotros decimos a los usuarios que no trabajamos con esto. Lo aceptan, porque no hay más. Decimos: «No trabajamos con esto, no trabajamos con esto». Yo creo que hay otros apoyos en la red que les pueden ofrecer alimentación. Puede ser Cáritas, Cruz Roja o los evangelistas. (Carla, E. I.)

El hecho de que una minoría de las profesionales pueda llevar a cabo semejante restricción de recursos sin (aparentes) injerencias por parte de las colegas o de otros niveles de la organización, y ante la indefensión de las usuarias, muestra el carácter altamente graciable que el apoyo institucional puede adquirir en un sistema de bienestar familista.

Ciertamente, el contexto institucional promueve que una parte de la población recientemente empobrecida no acuda a los servicios sociales (Enciso et al., 2016), posiblemente porque conciben que la asistencia social pública es residual, insuficiente, estigmatizadora e intrusiva. La siguiente cita muestra que ser usuaria de los servicios sociales básicos pueden percibirse como una situación que conlleve unos costes en términos de estatus e incluso de privacidad que no compensen las ayudas económicas (Paugam, 2002).

DANIELA: Creo que queda mucha [gente] que ha continuado sin venir [a servicios sociales], que ha tirado de familia, lo ha pasado fatal, y no ha venido a servicios sociales porque son "servicios sociales».

ANDREA: Y porque lo que obtendrás de ese espacio tampoco te resolverá la vida. Total que te den una ayuda de urgencia, que puedas comer una semana, no vale la pena...

Teresa: Y explicar mis penas a un tercero, por muy profesional que sea... Pues no. Esto es una barrera.

La asistencia social pública en un contexto familista se fundamenta en una selección de los beneficiarios que requiere mecanismos de medición de los recursos de las familias (means test). Estos mecanismos no funcionan debido a la ineficacia administrativa que muestran los países del sur de Europa (Moreno, 2000), así como al carácter inestable e informal del apoyo familiar. En la práctica, los medios de comprobación de recursos no son eficaces y pueden humillar a las usuarias. Por ejemplo, las profesionales revisan de forma rutinaria extractos bancarios y tiques de los supermercados de las usuarias con el fin de controlar sus ingresos y la utilización de las prestaciones (Sánchez, en prensa).

Por otro lado, la estrategia de movilización de los recursos de la red familiar que promueve el familismo económico puede integrarse con las orientaciones 
hacia la activación y la responsabilización individual que impregnan la asistencia social pública (Sánchez, 2019a). De esta forma, otra práctica degradante que ha sido normalizada en los servicios sociales básicos es la de exigir que las usuarias beneficiarias de prestaciones económicas demuestren su predisposición a obtener un empleo a través de mostrar a la profesional una hoja con las firmas de cada una de las empresas donde han dejado el curriculum vitae.

Las relaciones familiares y comunitarias son fuente tanto de recursos materiales y económicos como de «reconocimiento social» (Vidal, 2008). Por tanto, una protección institucional que proteja y fortalezca las esferas familiares y comunitarias es necesaria para contrarrestar los procesos contemporáneos de precarización social, que se caracterizan por combinar la exclusión laboral con el aislamiento social (Castel, 1995). Sin embargo, en la práctica cotidiana de la asistencia social pública de Tarragona, la hegemonía familista acaba provocando una concepción de la inclusión sesgada, donde la familia se presenta como un sistema infalible de integración social y bienestar.

En este sentido, el mantenimiento de las contradicciones entre los supuestos familistas de intervención y las duras realidades familiares y comunitarias de las usuarias requiere un "trabajo ideológico» (ideological work) (Morgen et al., 2009). Para ello, el contexto más amplio proporciona narrativas que legitiman las prácticas familistas en los servicios sociales básicos: «Savater dice que la "ONG familia" ha impedido un estallido social mayor en España" (eldiario.es, 26 de enero de 2013). De este modo, las profesionales podrían seleccionar — de forma no consciente- experiencias anecdóticas para crearse opiniones que, a la postre, le permitan resolver las dificultades prácticas y éticas de la gestión de la pobreza en un marco organizativo constrictivo:

Hay unas redes sociales montadas impresionantes. Podría hablar de nigerianos; ellos dicen: «No te preocupes, el hijo ya me lo cuidará esta». Y cuando vas a casa de alguno de ellos, siempre hay gente. (Liliana, G. F.)

\subsection{Dinámicas de contrafamilismo en los servicios sociales}

La función de asistencia social se entreteje en la práctica con otras funciones que los servicios sociales básicos también tienen atribuidas, como pueden ser la promoción y la supervisión de las "habilidades parentales», o el apoyo a las mujeres que han sido víctimas de violencia de género. La siguiente cita expone cómo un enfoque familista para resolver las dificultades económicas de una familia monoparental puede terminar por menoscabar el mandato institucional de capacitar a la usuaria en su rol de madre:

Si el niño depende de mamá, cómo la mamá solucione las cosas no es asunto mío. Una mamá que se organiza para delegar funciones, para que la ayuden, que tiene a la madre que le paga la guardería, y la suegra que le hace de canguro para ella poder hacer tres escaleras y un curso, ¡chapó! [...] El rollo es cuando el abuelo dice: «Tu madre es responsabilidad mía, y como ella es mi responsabilidad, ¡tú también!». Porque llegamos al punto de que el abuelo dice: «El 
niño no hará esta actividad porque el que paga soy yo, y la madre ya puede decir misa». (Beatriz, E. I.)

En ocasiones, el principio familista de conocimiento o de valoración de la situación familiar para la obtención de una prestación puede quedar supeditado a la decisión del personal de respetar la privacidad y los equilibrios internos de las familias. La siguiente cita justifica esta posible tramitación de una prestación económica sin comprobar la situación o la actitud hacia el empleo de la esposa del usuario que realiza la demanda:

No lo fuerzo más porque me parece demasiado invasivo [respecto a conocer a la esposa del demandante de la ayuda de urgencia]. Y es el marido que le lleva el papel a la mujer para que lo firme. Y tampoco decido hacer visita a domicilio. Creo que se debe respetar. Yo mantengo el límite de la relación. Entro si me dejan entrar. Quizás aquí debería ser más atrevida, para que ella vea que no pasa nada, que estamos para dar apoyo. Pero eso me cuesta. A mí, me cuesta. Supongo que yo quiero que respeten mucho mi privacidad, y eso lo trasladas al trabajo, porque son tus patrones de conducta normales. (Julia, E. I.)

Transformaciones legislativas y sociales han propiciado que los principios y las prácticas de igualdad de género formen parte del contexto institucional de los servicios sociales básicos. ${ }^{7}$ De esta manera, la protección de usuarias de sus parejas violentas, o de otras situaciones menos explícitas de opresión, configura prácticas institucionales que podrían erosionar los principios familistas tradicionales del sistema de bienestar en España. A continuación, se muestra una intervención social que prioriza la seguridad y la emancipación de la usuaria sobre la defensa de un núcleo familiar de tipo tradicional.

En casos donde no hay denuncia, hacemos un seguimiento. Con esta mujer se trata de hacer un acompañamiento de qué quiere, qué necesita y hasta dónde puede llegar. Es una chica joven que no ama a su pareja, pero que es su familia. Y es lo que hay. Hacerle ver que ella es mujer, que no todo es familia, y que la familia también puede ser mamá y niños, no siempre papá, mamá..., que se puede concebir de otras maneras. (Blanca, E. I.)

En todo caso, resulta importante señalar que las mujeres empobrecidas tienen más riesgos de sufrir violencia de género (Damonti, 2019). Por tanto, la falta de recursos de servicios sociales para que estas mujeres (a menudo, con hijos pequeños) puedan retomar nuevos proyectos de vida podría empujarlas a permanecer en situaciones extremas de riesgo y de sufrimiento. Por otra parte, la intervención desde servicios sociales en contra de la violencia de género también incluye principios familistas, porque (como tantas otras prácticas institucionales) se centra en (o pesa sobre) la mujer. En última instancia, la falta

7. Ley Orgánica 1/2004, de 28 de diciembre, de Medidas de Protección Integral contra la Violencia de Género. 
de iniciativas y de recursos eficaces dirigidos a la contención o reforma de los hombres maltratadores permite que la institución configure a estas exparejas como una amenaza constante de la que las mujeres deben ponerse a salvo.

Por otro lado, el familismo institucional, que instrumentaliza la familia extensa como un potencial recurso económico, convive con una concepción profesional de las «dificultades sociales» que, en buena medida, se explica por las «dinámicas familiares» (Fernández, 2015). En ocasiones, este principio orientador de la intervención social puede desafiar la hegemonía familista porque puede considerar que las dificultades de las usuarias se deben en parte a sus relaciones familiares. Sin embargo, esta aproximación puede, en última instancia, resultar condescendiente y silenciar las condiciones de desigualdad y de opresión.

También intento ponerme en su lugar. Quizás en su situación yo haría lo mismo. Si yo estoy aquí ha sido por las experiencias que he tenido. O por la gente que me rodea. Pero si esta familia no ha tenido nunca esta oportunidad de estar con alguien y siempre ha vivido en un mismo círculo, con la misma dinámica, es fácil que esté así. (Mónica, E. I.)

\subsection{Prácticas emergentes de familismo relacional}

La crisis reciente ha perjudicado más directamente a las familias con hijos pequeños (Marí-Klose, P. y Marí-Klose, M., 2015). Como se ha visto, los servicios sociales han instrumentalizado este sesgo generacional de la pobreza a partir de un familismo que promueve que madres, padres e hijos reciban apoyo económico de los abuelos. Sin embargo, desde primera línea de intervención se admite que esta estrategia no es sostenible porque eventualmente sobrecarga la red familiar. La práctica cotidiana de los servicios sociales puede desvelar un desajuste cada vez mayor entre la expectativa institucional del familismo económico y la nueva realidad socioeconómica e ideológica de un familismo relacional. Cada vez parece más difícil que la familia extensa resulte eficaz como última red de protección material. En cambio, resulta más factible reorientar las intervenciones familiares hacia la promoción de los vínculos afectivos y de intercambio de algunos favores:

EnTREVISTADOR: ¿¿Se prioriza el apoyo de la familia extensa si está en condiciones de ayudar?

Ariadna: Sí, claro, solo que la familia extensa últimamente ya no... La familia extensa es más de apoyo, de apoyo emocional, de saber que en un momento dado no te quedarás en la calle. [...] El núcleo familiar eres tú, eso no se puede olvidar. Quizás es más apoyo del tipo: «Tú vas a trabajar y el abuelo se queda con el niño». Esto a veces incluso les rejuvenece. (E. I.)

Por otra parte, esta redefinición del familismo hacia su dimensión más relacional podría ampliar su alcance hasta el punto de cuestionar que la con- 
sanguineidad deba ser el principio definidor de la solidaridad familiar. De esta forma, emergen formas institucionales de promoción de intensas redes de apoyo entre usuarias que mantienen identidades sociales similares o que se encuentran en situaciones parecidas:

La familia son las amigas que hacen entre ellas [madres víctimas de violencia de género]. Yo tengo tres así, una del Pakistán, otra nigeriana y otra de Irán. Entre la del Pakistán y la de Irán hay complicidad, hablan el mismo idioma. Hago reuniones con las tres y charlamos todas juntas. [...] Cuando una se pone mala, pues les llama para que vayan a buscar al niño. Si encima no tuvieran estas amigas, no sé cómo se lo harían. (Carla, E. I.)

\section{Discusión de resultados}

Este artículo ha mostrado que el familismo institucional mantiene una fuerte base material (falta de prestaciones) e ideológica (la familia como capaz y responsable de la protección económica de sus miembros) que condiciona y, en ocasiones, desfigura las acciones contra la pobreza de los servicios sociales. La institución tiende a sobreestimar la capacidad inclusiva de la familia extensa actual, a veces en detrimento del bienestar y de las opciones de la usuaria. Por ejemplo, a las jóvenes madres sin recursos propios a menudo no se les propone otra alternativa que la de retornar al hogar de los padres. Asimismo, esta lógica familista en la implementación de las prestaciones puede proyectarse sin reservas en las relaciones de las usuarias con sus compatriotas o con sus vecinos. El recurso sistemático a estas fuentes informales de bienestar conlleva el riesgo de enmarañar a las usuarias en redes de desventaja social. Asimismo, los procedimientos de la selección de personas beneficiarias de prestaciones en un marco familista pueden acentuar los procesos de estigmatización de la población más precarizada.

Por otra parte, desde servicios sociales se puede revertir, en parte, el supuesto familista cuando predominan otras influencias institucionales que orientan la intervención a la protección de la infancia o la autonomía de la mujer. No obstante, la falta de recursos efectivos para implementar prácticas emancipadoras y una orientación del personal que tiende a explicar las dificultades sociales por la situación familiar reducen la eficacia de los servicios sociales básicos. Finalmente, existen dinámicas incipientes o minoritarias de redefinición institucional de la lógica familista que pueden favorecer la inclusión social y, de forma indirecta, el bienestar económico de las familias empobrecidas. Primero, los servicios sociales pueden promover la expectativa familista de apoyo emocional y de favores entre parientes para contrarrestar procesos emergentes de fragilidad relacional. Segundo, esta orientación menos materialista de los vínculos familiares permite a las trabajadoras y educadoras sociales constituir como familias grupos de usuarias en situación de vulnerabilidad relacional a partir de la cohesión que producen las afinidades personales o el hecho de pasar por una misma situación vital. 


\section{Conclusiones}

El sistema de bienestar del sur de Europa se caracteriza por mantener una complementariedad (precaria) entre unas políticas de protección social residuales y los sistemas familiares y comunitarios de ayuda mutua («subsidiariedad pasiva de las políticas sociales») (Garcia y Kazepov, 2002). Este artículo muestra que esta interrelación entre el sistema institucional y estos recursos de bienestar informales no siempre se produce de forma automática sino que puede requerir de las prácticas reflexivas de las profesionales de los servicios sociales básicos. El análisis también identifica los límites y apunta a las posibles reconfiguraciones del familismo en el modelo de bienestar de la España actual.

Efectivamente, las condiciones institucionales de los servicios sociales implican que la solidaridad familiar continue siendo el principal recurso de la asistencia social pública. No obstante, las intervenciones familistas resultan a menudo ineficaces porque el "capital social» de las familias empobrecidas carece en sí mismo de capacidad económica, cultural y de legitimación social (Bourdieu, 1980). La evidente ineficacia de las redes familiares para proteger numerosos hogares empobrecidos apunta a la posibilidad de que este familismo institucional persista en parte por la necesidad de mantener el orden social (Aguilar, 2010). Sin embargo, la urgencia y la complejidad de las necesidades que se personifican a diario en los servicios sociales básicos actuales lleva a las profesionales a producir nuevas prácticas que redefinen algunos supuestos familistas. Actualmente, los apoyos emocionales y de favores no monetarios de la familia extensa pueden resultar un recurso de bienestar más factible que el apoyo económico directo. Además, el carácter polifacético de las situaciones de desventaja y también la influencia de otros principios de intervención en los servicios sociales problematizan la aplicación sistemática del supuesto de que la familia extensa debe socorrer económicamente los hogares empobrecidos, sobre todo cuando esto puede perjudicar la seguridad, el bienestar o la dignidad de las usuarias.

Mientras que la exclusión relacional empobrece a las personas, no parece que la mera participación en redes sociales permita escapar de la precariedad económica (Afrida, 2011). En este sentido, el presente estudio confirma la necesidad de que se establezcan prestaciones mínimas garantizadas para satisfacer las necesidades básicas de los hogares empobrecidos y prevenir el desgaste de las redes familiares (Martínez, 2014). En un marco de prestaciones garantizadas, los servicios sociales pueden promover una integración social basada en el bienestar y las oportunidades de las usuarias, y no en la desesperación que comporta la privación material extrema. En este sentido, los servicios sociales se deben configurar como un capital institucional de las familias empobrecidas que les ayude a identificar y conectar con nuevas relaciones familiares y comunitarias. En ocasiones, estos hogares requerirán intervenciones previas que les proporcionen habilidades y recursos que les capaciten en el acceso a dinámicas de reciprocidad. En todo caso, las intervenciones deben incorporar la perspec- 
tiva de género para revertir la tendencia de muchas relaciones sociales a ser más exigentes con las mujeres. De esta forma, el acceso de los hogares a relaciones positivas con su entorno debe considerarse un objetivo de las políticas sociales y no tanto un supuesto sobre el que delegar la intervención.

En definitiva, este artículo demuestra que la asistencia social pública en España no consiste simplemente en una política social residual, que favorece los procesos de refamilización en momentos de crisis económica. Ciertamente, la práctica de los servicios sociales básicos puede redefinir los contornos y la sustancia de la solidaridad familiar, dentro de un contexto más amplio de transformación de la institución familiar. En última instancia, el carácter más o menos inclusivo de las nuevas formas de solidaridad que las instituciones entretejan dependerá de la dotación de recursos económicos, y de la consolidación de un marco institucional e ideológico que priorice el bienestar y las oportunidades de las personas.

\section{Referencias bibliográficas}

Adelantado, José y Noguera, Antonio (1999). «Reflexionando sobre las relaciones entre política social y estructura social». Papers: Revista de Sociologia, 59, 71-77. $<$ https://doi.org/10.5565/rev/papers/v59n0.1256>

Afrida, Asif (2011). Social networks: their role in addressing poverty. York: Joseph Rowntree Foundation.

Aguilar, Manuel (2010). «La huella de la beneficencia en los Servicios Sociales». Zerbituan, 48, 9-48. <https://doi.org/10.5209/revCUTS.2013.v26.n1.41664>

- (2013). «Los servicios sociales en la tormenta». Documentación Social, 166, 145-167

Aguilar, Manuel; Llobet, Marta y Pérez, Begoña (2012). «Los servicios sociales frente a la exclusión». Zerbitzuan, 5, 9-26. $<$ https://doi.org/10.5569/1134-7147/51.01>

Andreotti, Alberta; García, Marisol; Gómez, Aitor; Hespania, Pedro; Kazepov, Yuri y Mingione, Enzo (2001). «Does a Southern European Model Exist?». Journal of European Area Studies, 9 (1), 43-62. $<$ https://doi.org/10.1080/14608460120061948>

Andreotti, Alberta; Mingione, Enzo y Polizzi, Emanuele (2012). «Local Welfare Systems: A Challenge for Social Cohesion». Urban Studies, 49 (9), 1.925-1.940. $<$ https://doi.org/10.1177/0042098012444884>

Arriba, Ana y Moreno, Luis (2005). «Spain. Poverty, social exclusion and "safety nets"», 141-203. En: Ferrera, M. (ed.). Welfare State Reform in Southern Europe: Fighting poverty and social exclusion in Italy, Spain, Portugal and Greece. Londres: Routledge.

Bazo, Teresa (2012). «Relaciones familiares y solidaridad intergeneracional en las nuevas sociedades envejecidas". Panorama Social, 15, 127-142. $<$ https://doi.org/10.1017/S0144686X100009>

Beck, Ulrich; Giddens, Anthony y Lash, Scott (1994). Reflexive Modernization. Politics, Tradition and Aesthetics in the Modern Social Order. Cambridge: Polity Press.

Becker, Howard; Geer, Blanche; Hughes, Everett y Strauss, Aselm (1961). Boys in White: Student Culture in Medical School. Chicago: University of Chicago Press. 
Bertaux, Daniel (2010). Le récit de vie. L'enquête et ses méthodes. París: Armand Colin. Bourdieu, Pierre (1980). "Le capital social. Notes provisoires». Actes de la Recherche en Sciences Sociales, 31, 2-3.

Castel, Robert (1995). Les métamorphoses de la question sociale: une chronique du salariat. París: Gallimard.

Comisión Europea (2008). Child Poverty and Well-Being in the EU Current Status and Way Forward. Luxemburgo: Publications Office of the European Union.

Consejo General del Trabajo Social (2012). Código Deontológico del Trabajo Social. Consulta 25 de julio de 2019. Disponible en <http://www.cgtrabajosocial. es/codigo_deontologico>.

Damonti, Paola (2019). «Exclusión social como factor de riesgo de violencia de género en la pareja». Papers: Revista de Sociologia, 104 (3), 485-523. $<$ https://doi.org/10.5565/rev/papers.2570>

Déchaux, Jean (2007). «Réalités et limites de l'entraide familiale». En: Paugam, Serge (dir.). Repenser la solidarité. L'apport des sciences. París: UPF.

ECAS (2012). Propostes i reflexions al voltant del programa de Renda Minima d'Inserció. Barcelona: ECAS.

eldiario.es (26 de enero de 2013). «Savater dice que la "ONG familia” ha impedido un estallido social mayor en España».

Enciso, Joan; Allepuz, Rafa; Lapresta, Cecilio y Pelegrí, Xavier (2016). «Nueva y vieja pobreza». Praxis Sociológica, 20, 93-112. <https://doi.org/10.3989/ris.2010.11.22>

Esping-Andersen, Gosta (1999). Social foundations of postindustrial economies. Oxford, Nueva York: Oxford University Press.

- (2002). Why We Need a New Welfare State. Oxford: Oxford University Press.

Fantova, Fernando (2008). Sistema público de servicios sociales. Nuevos derechos, nuevas respuestas. Bilbao: Publicaciones de la Universidad de Deusto.

FERnÁndeZ, Josefa (ed.) (2015). La intervención con las familias desde el trabajo social. Barcelona: Universitat de Barcelona.

Flaquer, Lluís (1998). El destino de la familia. Barcelona: Ariel.

- (2004). «La articulación entre familia y el Estado de bienestar en los países de la Europa del sur». Papers: Revista de Sociologia, 73, 27-58. <https://doi.org/10.5565/rev/papers/v73n0.1105>

- (2010). «Famílies i relacions familiars». Revista Papers. Institut d'Estudis Regionals i Metropolitans de Barcelona, 52, 50-62.

Flaquer, Lluís; Almeda, Elisabet y Navarro-Varas, Lara (2006). Monoparentalidad e infancia. Barcelona: Fundación La Caixa.

Garcia, Marisol y Kazepov, Yuri (2002). "Why some people are more likely to be on social assistance than others». En: SARACEno, Chiara. Social assistance dynamics in Europe. National and local poverty regimes. Bristol: The Policy Press.

González, Sergio y Pino, Eloisa del (2017). From letting Europe into policy conditionality. Welfare reform in Spain under austerity. Madrid: Working Papers 1701. Instituto de Políticas y Bienes Públicos (IPP-CSIC).

Izaola, Amaya y Zubero, Imanol (2015). «La cuestión del otro: forasteros, extranjeros, extraños y monstruos». Papers: Revista de Sociologia, 100 (1), 105-129. <https://doi.org/10.5565/rev/papers.649>

IVALUA (2016). Avaluació del Fons d'ajuts d'emergència social per a infants menors de 16 anys. Informe definitiu. Barcelona: IVAULA. 
Lasheras, Rubén; Martínez, Lucía y Azcona, Patricia (2012). "Condiciones de vida, estrategias e itinerarios de exclusión de los hogares en Navarra». En: CorERA, Jesús; Laparra, Miguel y Pérez-Eransus, Begoña (coord.). Informe sobre el impacto de la crisis en los hogares en situación de exclusión en Navarra. Villatuerta: Gráficas Lizarra.

Lenoir, Rémi (2007). «La solidarité familiale: une question morale?». En: PAugam, Serge (dir.). Repenser la solidarité. L'apport des sciences sociales. París: Presses Universitaires de France.

Lipsky, Michael (1980). Street-level Bureaucracy: Dilemmas of the Individual in Public Services. Nueva York: Russell Sage Foundation.

Lister, Ruth (2004). Poverty. Cambridge: Polity Press.

Llano, Juan Carlos (2019). El estado de la pobreza. Seguimiento del indicador de pobreza y exclusión social en España 2008-2018. Madrid: EAPN.

Marí-Klose, Marga (2016). "Solidaridad intergeneracional en época de crisis: ¿mito o realidad?». Panorama Social, 22, 61-78.

Marí-Klose, Pau y Marí-Klose, Marga (2015). «Políticas de protección social contra la pobreza infantil mediante transferencias monetarias». Indice: Revista de Estadistica y Sociedad, 63, 38-40.

Martínez, Lucía (2014). Sintomas de agotamiento de la solidaridad familiar. Madrid: Foessa.

McCave, Angus; Gilchist, Allison; Harri, Kevin; Afridi, Asif y Kyprianou, Pau (2013). Making the links: poverty, ethnicity and social networks. York: Joseph Rowntree Foundation.

MeIL, Gerardo (2011). Individualización y solidaridad familiar. Barcelona: Obra Social La Caixa.

Moreno, Luis (2000). Ciudadanos precarios. La «última red» de protección social. Barcelona: Ariel.

- (2001). "La "vía media" española del modelo de bienestar mediterráneo». Papers: Revista de Sociologia, 63-64, 67-82. <https://doi.org/10.5565/rev/papers/v63n0.1207>

Moreno Mínguez, Almudena (2012). «La invisibilidad de los y las jóvenes en los servicios sociales del Estado de bienestar español». Revista de Estudios de Juventud, 97, 93-108.

Morgan, David (1996). «Focus Groups». Annual Review of Sociology, 22, 129-152. <https://doi.org/10.1146/annurev.soc.22.1.129>

Morgen, Sandra; Acker, Joan y Weight, Jill (2009). Stretched Thin: Poor Families, Welfare Work, and Welfare Reform. Cornell University Press.

OBJOVI (2013). «Informe del Observatorio de Emancipación elaborado por el Consejo de la Juventud de España». Obtenido de <http://www.cje.org/descargas/cje5225.pdfs.

OECD (2011). Doing Better for Families. París. OECD Publishing.

Parella, Sonia (2000). «El trasvase de desigualdades de clase y etnia entre mujeres». Papers: Revista de Sociologia, 60, 275-289. <https://doi.org/10.5565/rev/papers/v60n0.1043>

Paugam, Serge (2002). La disqualification sociale. París: La Decouverta.

- (2005). Les formes élémentaires de la pauvreté. París: Le Lien Social.

Portes, Alejandro (1998). «Social Capital: Its Origins and Applications in Modern Sociology». Annual Review of Sociology, 24, 1-24. <https://doi.org/10.1146/annurev.soc.24.1.1>

Power, Ann; Willmot, Hellen y Davidson, Rosemary (2011). Family futures. Childhood and poverty in urban neighbourhoods. Bristol: Policy Press. 
Raitano, Michele (2015). «Intergenerational Transmission of Inequalities in Southern European Countries in Comparative Perspective: Evidence from EU-SILC 2011». European Journal of Social Security, 17 (2), 272-292. <https://doi.org/10.1177/138826271501700208>

Ridge, Tess (2010). Living with poverty. A review of the literature on children's and families' experiences of poverty. Londres: Department for Work and Pensions.

Sánchez, Sergio (en prensa). El trabajo social en acción. Prácticas profesionales en los servicios sociales básicos. Barcelona: Colegio Oficial de Trabajo Social de Cataluña.

- (2019a). «Social workers implementing social assistance in Spain: reshaping poverty in a familialistic welfare state», 169-183. En: Ute KLAmmer, U.; Leiber, S. y LeitNER, S. (ed.). Social Work and the Making of Social Policy. Bristol: The Policy Press. <https://doi.org/10.2307/j.ctvhktj6v.16>

- (2019b). «Social capital and poverty in a Southern-European urban context». Autonomie Locali e Servizi Sociali, 1, 89-104. <https://doi.org/10.1447/95338>

Saraceno, Chiara (1995). «Familismo ambivalente y clientelismo categórico en el Estado del bienestar italiano». En: Sarasa, Sebastián y Moreno, Luis (eds.). El Estado del bienestar en la Europa del sur. Madrid: Instituto de Estudios Sociales Avanzados.

- (2002). Social assistance dynamics in Europe. National and local poverty regimes. Bristol: The Policy Press.

Sieben, Barbara y Wettergren, Åsa (ed.) (2010). Emotionalizing Organizations and Organizing Emotions. Basingstoke: Palgrave Macmillan.

Silverman, David (2010). Doing Qualitative Research. Londres: SAGE Publications.

Subirats, Joan (dir.) (2007). Los servicios sociales de atención primaria ante el cambio social. Madrid: Ministerio de Trabajo y Asuntos Sociales.

Tönnies, Ferdinand (1984 [1887]). Comunitat i associació. Barcelona: Edicions 62.

Van Berkel, Rik y VAn der Aa, Paul (2012). "Activation work: Policy programme administration of professional service provision?». Journal of Social Policy and Politics, 41, 493-510. <https://doi.org/10.1017/S0047279412000062>

VidAL, Fernando (coord.) (2008). «Capital social y capital simbólico como factores de exclusión y desarrollo social». En: VI Informe sobre exclusión y desarrollo social en España 2008, 525-598. Madrid: Cáritas.

Vinzant, Janet y Crothers, Lane (1996). «Street-level Leadership: Rethinking the Role of Public Servants in Contemporary Governance». American Review of Public Administration, 26, 4, 457-475. <https://doi.org/10.1093/oxfordhb/9780199226443.003.0020>

Williams, Colin y Windebank, Jan (2000). «Self-help and Mutual Aid in Deprived Urban Neighbourhoods: Some Lessons from Southampton». Urban Studies, 37, $127-147$. <https://doi.org/10.1080/0042098002320>

Yin, Robert (2014). Case study research. Design and methods. Los Angeles: Sage. 